\title{
Self efficacy measurement and goal attainment after pulmonary rehabilitation
}

\author{
Rachel Garrod \\ Johanna Marshall \\ Fiona Jones \\ School of Physiotherapy, St George's, \\ University of London and Kingston \\ University Faculty of Health and \\ Social Care Sciences, UK
}

\begin{abstract}
Background: Little is known about self-efficacy in COPD and its role as a mediator of goal attainment after rehabilitation. We hypothesized that higher baseline self efficacy may facilitate goal attainment.
\end{abstract}

Methods: 48 COPD patients completed pulmonary rehabilitation, self-efficacy was measured using the COPD Self Efficacy Scale (CSES). Personal goals were used as self reported outcome. Relationships were evaluated between CSES and St George's Hospital Respiratory Questionnaire (SGRQ); depression; using Brief Assessment Schedule Cards (BASDEC), London Chest Activity of Daily Living Scale (LCADL) and exercise tolerance; using Six-Minute Walking Distance (6MWD) and muscle strength.

Results: 74 Stable COPD patients, mean $\mathrm{FEV}_{1} 1.2$ (0.6) 1, age 68.1 (10.2) years were recruited. 51 patients completed rehabilitation and 48 of those CSES and reported goal attainment. 94 goals were documented. Baseline self efficacy did not differ according to whether goal was achieved or not. Relationships were evident between CSES and SGRQ $(r=-0.53), 6 \mathrm{MWD}(\mathrm{r}=0.36)$, $\operatorname{BASDEC}(\mathrm{r}=-0.31)$, LCADL $(\mathrm{r}=-0.33)($ all $\mathrm{p} \leq 0.01)$, but not $\mathrm{FEV}_{1}$, pack years or muscle strength. There was a significant improvement in CSES scores pre to post rehabilitation, mean difference (95\% CI) 0.27 (0.04 to 0.51$)$.

Conclusions: Self efficacy, using the CSES, improves with rehabilitation but baseline self efficacy does not appear to influence goal attainment.

Keywords: self-efficacy, COPD, pulmonary rehabilitation, goal

\section{Introduction}

Chronic Obstructive Pulmonary Disease (COPD), characterized by fixed airflow obstruction, results in significant morbidity and mortality. It is the third greatest cause of respiratory death in the United Kingdom with older people increasingly affected (British Thoracic Society 2006). Patients suffer impairment in health related quality of life (HRQL) distressing dyspnoea and reduced exercise capacity (National Collaborating Centre for Chronic Conditions. 2004). COPD accounts for $20 \%$ of all respiratory related emergency admissions to English hospitals (British Thoracic Society 2006). Chronic diseases such as COPD require a holistic approach to management, and importantly a strong focus on the facilitation and support of behaviors that enable selfmanagement. Pulmonary rehabilitation, a multidisciplinary intervention consisting of exercise, psycho-social support and education aims to "reduce the symptoms, disability, and handicap and to improve functional independence in people with lung disease" (Morgan et al 2001). In addition to this pulmonary rehabilitation encourages a commitment to long term exercise behaviors and promotes self-management strategies.

Self efficacy is a psychological construct which defines "the belief in one's capabilities to organise and execute the course of action required to produce given attainments" (Bandura 1997), and may be fundamental to promoting effective self management and enabling behavior change in the longer term (Jones 2006). In one study of healthy older subjects, self efficacy was shown to be influential in an individual's decision concerning
Correspondence: Rachel Garrod

Tel +4402087250377

Fax +44 02087252248

Email rgarrod@hscs.sgul.ac.uk 
participation in a home-based strengthening programme (Bawel 2003). It has been suggested that self-efficacy acts as the mediator between changes in health related quality of life, symptoms and physiological outcomes in patients with COPD after pulmonary rehabilitation (Arnold et al 2006; Kohler 2002). In support of this, change in exercise tolerance after rehabilitation has been shown to be associated with improvements in self-efficacy (Lox and Freehill 1999). In this case the self-efficacy measurement was designed to test an individual's confidence to perform selected activities as extolled by Bandura (1997). Participants were asked to rate perceived confidence to achieve pre-defined walking distances. The authors demonstrated that stronger self-efficacy for walking at the start of the programme was associated with a greater increase in walking distance (Lox and Freehill 1999). This suggests a role for confidence and perceived ability in achieving change. In another study of individuals with COPD the authors evaluated relationships between selfefficacy, dyspnoea and functional ability (Siela 2003). Using the COPD Self Efficacy Scale (CSES) significant relationships between the variables were identified. Self-efficacy accounted for one third of the variance in functional ability in men with COPD (Siela 2003). The CSES was developed specifically for COPD and has shown good test-retest reliability and internal consistency (Wigal et al 1991) although to date the scale has received little attention in the evaluation of pulmonary rehabilitation.

Goal setting is commonplace in many programmes, and often considered to be "the cornerstone of effective rehabilitation“ (Barnes and Ward 2000). Effective goal setting and attainment of goals is generally thought to be associated with increased confidence in self-management skills, for example the ability to manage symptoms and self-exercise. The precise value and effectiveness of methods of goal setting has, however been poorly evaluated (Levack et al 2006) and factors that determine those who achieve goals compared with those who do not requires greater exploration. One study using 'goals achieved' as a measurement of success identified significant differences in the assessment of goal attainment between therapists and patients. Therapists were more likely to underestimate goal attainment compared with patients (van Stel et al 2002). We may hypothesise that self-efficacy will determine whether a patient develops the necessary behaviors to achieve a particular goal. The aims of this study therefore are to investigate the influence of initial levels of self-efficacy on patient reported goal achievement and the effect of rehabilitation on self efficacy. In addition, we will explore relationships between self efficacy and other rehabilitation outcomes.

\section{Methods}

In this longitudinal follow up study, patients were recruited from primary and secondary care services as part of a study of pulmonary rehabilitation (Garrod et al 2006). One hundred and eleven patients with a known diagnosis of COPD (The COPD Guidelines Group of the Standards of Care Committee of the British Thoracic Society 1999) were sent invitation letters outlining the rehabilitation programme on offer and the nature of the research. Of those who responded, 16 declined to participate and 87 made initial assessment appointments. Seventy-four of these patients attended initial assessment (missing CSES data for 2 patients). All provided written informed consent. All patients had limited exercise tolerance due to dyspnoea. No patient had performed rehabilitation in the past 12 months. Taxi transport to the hospital was provided where required. Exclusion criteria consisted of unstable angina, recent exacerbation or change of medication, intermittent claudication or other mobility limiting conditions. Full ethical consent was obtained from Wandsworth Local Research Ethics Committee.

\section{Baseline assessments}

Patient details (age, gender, smoking history) and lung function $\left(\mathrm{FEV}_{1}\right.$ and $\mathrm{FVC}$ ) were assessed prior to rehabilitation using a Sensormatics ${ }^{\mathrm{TM}}$ Vmax $29 \mathrm{c}$.

\section{Goal setting}

Goal setting was performed prior to rehabilitation with the assistance of the physiotherapist. The patient first described the goal and therapy input was provided in order to ensure that goals were measurable and realistic. Goals were written in the patient case files by the therapist and accuracy confirmed with the patient. Goals were set with the intention of use as a subjective patient reported outcome measure (Levack et al 2006). All patients identified at least 1 goal, patients were encouraged to set more goals, up to a maximum of 3 , as appropriate. Goals were not ordered according to importance. The following are examples of goals set;

"I want to be able to get to the library again"

"To be able to walk from ....Hill to the end of ...... Road with $50 \%$ less breathlessness"

"Would like to be able to do some creative work again eg; make models/saw wood.

"To be able to go up and down flight of stairs at home with the oxygen on my own (currently needing assistance of 1)"

"To be able to bath without assistance"

"I want to be able to walk to corner shop at same speed as husband and continue a conversation". 
The following outcomes were recorded prior to and post rehabilitation:

\section{Goal attainment}

Goals were scored after rehabilitation using the criteria "fully achieved", "half achieved", "not achieved". Where there was doubt concerning achievement the goal was scored as "not achieved". Attainment of goal was patient determined since previous work has shown that therapists' assessment of goal attainment may under estimate patient perception of achievement (van Stel et al 2002).

\section{Primary outcomes Self efficacy}

This was assessed using the Chronic Obstructive Pulmonary Disease Self-Efficacy scale (CSES). This 34 item questionnaire has been shown to be both reliable and internally consistent (Wigal et al 1991). Participants are asked to identify how confident they are that they could manage their breathing difficulties in a number of situations, for instance "when there is humidity in the air", "when I am lying in bed". The CSES provides items with sufficient complexity in relation to the specific situation of managing with COPD. The CSES consists of Likert scale with 5 responses from "very confident" to "not at all confident" scoring 5 to 1 with 5 representing higher self-efficacy. The score can be calculated in two ways, a raw score with a maximum of 170 , or a rating score which takes account of items that may not be applicable to the patient by reporting the average score based on the number of items answered. Since, in some cases, items were considered non applicable, we only report on the rating score data in this analysis.

\section{Secondary outcomes}

\section{Sub-maximal exercise tolerance}

The Six Minute Walking Distance (6MWD) was performed according to ATS instructions (ATS 2002) along a $30 \mathrm{~m}$ corridor and the best of 2 repeatable tests were taken (repeatability was defined as a difference between walks of $<50 \mathrm{~m}$ ). Results of the 6MWD were recorded as actual values and as percent predicted (\% pred) of normative data (Enright and Sherrill 1998).

\section{Health status}

This was assessed using the St. George's Respiratory Questionnaire (SGRQ) (Jones et al 1992). This 50 item, disease-specific, self-complete questionnaire has been validated to measure health impairment in respiratory patients. Scores range from 100 'worst possible health status' and 0 'best possible health status.

\section{Quadriceps strength}

This was assessed using a Cybex Norm $^{\mathrm{T}}$ Testing and Rehabilitation System, in accordance with manufacturer's instructions. The equipment was set to test knee extension isokinetically, in seated mode, starting from full knee flexion. A speed of 60 degrees per second was selected. Maximum torque was recorded as the best of three attempts from the dominant side (Nms) and as Nms/subject bodyweight $(\mathrm{kg}) * 100$. Reliability and sensitivity has been previously demonstrated (Troosters, Gosselink, and Decramer 2001).

\section{Depression}

The Brief Assessment Schedule Depression Cards (BASDEC) were used to assess depression (Yohannes et al 2000). A score of 7 or above indicates possible depression.

\section{Breathlessness during daily activities}

This was assessed using the London Chest Activity of Daily Living Scale (LCADL), a 15 item questionnaire designed to measure dyspnoea during routine daily activities in patients with COPD. Higher scores represent maximal disability (Garrod et al 2002).

\section{The rehabilitation programme}

The rehabilitation programme was delivered over seven weeks, patients attended twice weekly as out-patients. Provision was made for additional attendance due to missed sessions. Each session comprised one hour of exercise followed by an education session. The exercise programme has been previously described (Garrod et al 2004) and was delivered in accordance with British Thoracic Society standards (Morgan et al 2001). In addition to attending class patients were asked to undertake a simple home exercise programme for approximately twenty minutes per day, five days per week. During the classes patients were reminded of their goals and encouraged to work towards them at home. Family members who attended were also informed of patient goals.

\section{Analyses}

All data were normally distributed. In order to consider all goals reported, a new aggregated measure was defined as "Attained" based on the fact that at least one goal was fully achieved. Where no goals were fully achieved the aggregated measure was defined as "Unattained". Unpaired t-test was used to examine difference between baseline self efficacy according to goal attainment. To identify whether 
self-efficacy changed with rehabilitation a paired t test was used on pre and post data. Relationships between baseline variables and self-efficacy were tested using Pearsons correlation co-efficient. All analyses were performed using SPSS 14 and significance was taken as $\mathrm{p}<0.05$.

\section{Results}

Of the 74 patients included in the study 51 (31 Male); mean \% pred FEV 1 (SD) 47.9 (22.6) \%; mean FEV 1 (SD) 1.17 (0.6) 1t; age (range) 68.5 (48-83), completed the rehabilitation programme and goals were scored on 48 of these (missing data on 3), CSES was missing on 1 patient who completed rehabilitation and goals. Additional details concerning baseline characteristics and severity of the patients have been previously reported (Garrod et al 2006).

\section{Self efficacy and goal attainment}

In total 96 goals were identified and reported on, (48 first goals, 41 second goals, 7 third goals) (Table 1 shows the breakdown of goals according to patient perception of achievement). Considering the aggregated measure, baseline self efficacy was not significantly different according to whether goals were "attained" or "unattained", mean (SD) $3.05(0.87)(\mathrm{n}=33)$ and $2.99(0.63)(\mathrm{n}=16)$ respectively $(\mathrm{p}=0.27)$.

\section{Change in CSES with pulmonary rehabilitation}

There was a significant improvement in CSES scores pre to post rehabilitation, mean difference $(95 \% \mathrm{CI}) 0.27$ (0.04-0.51).

\section{Drop outs}

We have previously reported comparisons between patients who dropped out of this study (and therefore did not achieve their goals) and those that completed the rehabilitation programme (Garrod, Marshall, Barley, and Jones 2006). Self efficacy at baseline did not differ between these groups and was not associated with drop out.

Table I Goal breakdown and achievement according to patient perception

\begin{tabular}{llll}
\hline & $\begin{array}{l}\text { Goal I } \\
(\mathbf{n = 4 8 )}\end{array}$ & $\begin{array}{l}\text { Goal 2 } \\
(\mathbf{n = 4 1 )}\end{array}$ & $\begin{array}{l}\text { Goal 3 } \\
(\mathbf{n = 7 )}\end{array}$ \\
\hline Fully achieved (\%) & 56.0 & 32.6 & 57.1 \\
Half achieved (\%) & 12.0 & 27.9 & 28.6 \\
Not achieved (\%) & 32.0 & 39.5 & 14.3 \\
\hline
\end{tabular}

\section{Relationships with CSES and baseline variables}

Statistically significant relationships were evident between CSES and baseline variables (Table 2), higher self-efficacy was associated with less depression, less breathlessness, higher health status and greater exercise tolerance. It was not associated with age, pack years, airflow obstruction or maximal quadriceps torque. Baseline self-efficacy did not differ according to gender.

\section{Discussion}

Pulmonary rehabilitation endeavors to facilitate and support long term behavior change in individuals with chronic disease. Education, self management strategies and goal setting (as components of rehabilitation) may be considered integral to this process. However the efficacy of methods of goal setting has not been clearly determined. Goal setting and problem solving may be considered an essential source of self-efficacy beliefs and relies on an individual's cognitive appraisal of the situation and effort to persist in the face of any setbacks. The findings of this study did not support our hypothesis, that individuals with strong self-efficacy would be more successful in overcoming difficulties in order to achieve their goals. In our analysis all goals were considered and classed as "attained" if at least one goal was fully achieved according to the patient's perspective. If data were analysed considering the primary goal only we see a trend emerging towards higher self efficacy associated with goal attainment $(\mathrm{p}=0.05)$. However, rehabilitation is

Table 2 Relationships between COPD Self-efficacy Scale (CSES) (Rating score) and baseline variables

\begin{tabular}{lll}
\hline $\mathbf{N}=72$ & \multicolumn{2}{l}{ CSES rating score $^{\mathrm{a}}$} \\
\cline { 2 - 3 } & $\mathbf{R}$ value & P value \\
\hline 6MWD & 0.37 & $<0.01$ \\
BASDEC & -0.31 & $<0.01$ \\
LCADL & -0.33 & $<0.01$ \\
SGRQ Total Score & -0.53 & $<0.00$ \\
Smoking pack years & -0.11 & 0.36 \\
Age (years) & 0.02 & 0.84 \\
Quadriceps maximal & -0.68 & 0.57 \\
torque (N/kg) & & \\
FEV & 0.22 & -0.59 \\
\hline
\end{tabular}

${ }^{a}$ CSES higher score $=$ greater self efficacy and higher BASDEC $=$ higher depression. LCADL Higher score $=$ worse breathlessness.

Abbreviations: 6MWD, 6 minute walking distance; BASDEC, the brief assessment schedule depression cards; LCADL, london chest activity of daily living scale*; SGRQ, st george's respiratory questionnaire; $\mathrm{FEV}_{1}$, forced expiratory volume in one second. 
a multifaceted therapy with the aim of achieving a number of goals relevant to patients' daily life, to this end we felt an aggregated measure would better reflect the complexity of rehabilitation and goal setting. It is however, reassuring that most of the patients reported some level of achievement of goals, hence supporting the notion that pulmonary rehabilitation is a patient focused treatment with outcomes directly applicable to patient goals.

The concept of goal setting remains poorly defined, not only do approaches differ between health professionals but individual concepts of goal setting and perception of attainment also differ. This makes standardization of goal setting difficult, however, we ensured that only one health professional was involved with the goal setting and that patients used a standardized method to record outcome, from their own perspective. Although goals necessarily differed according to patient preference we endeavored to ensure that they were measurable to some extent, eg, by distance walked, activity achieved, effort involved. The goals identified were predominantly related to functional ability suggesting there may have been (albeit unwitting) therapist bias. Further studies exploring the concept of goal setting in patients with COPD are recommended. In particular, the effect of supervision and the influence of different health professionals require further elucidation. The most appropriate way of measuring and assessing goals has yet to be determined and warrants further research (Levack et al 2006).

In this study we provide further validation of the CSES questionnaire through evidence of relationships with other measures of patient symptoms. Self-efficacy both influences whether an individual will attempt an action as well as determining whether they will persevere in overcoming obstacles (Bandura 1997). Self-efficacy beliefs have been found to predict significant variation in rehabilitation outcome following orthopedic surgery (after controlling for physical functioning) (Waldrop et al 2001), level of disability from pain intensity (Arnstein et al 1999) and recovery from heart attack (Ewart 1992).

A criticism of our study concerns the large number of subjects lost to follow-up. Of the initial 87 patients who made an appointment for assessment only 74 attended and CSES data were unavailable for 2 of those. Forty-eight patients completed rehabilitation and all assessments. Although self-efficacy at baseline did not differ between those who completed rehabilitation and those who dropped out we cannot account for differences in motivation nor do our analyses allow us to explore other psychological influences such as anxiety or depression that might contribute to goal attainment. However, whether baseline self efficacy influences goal attainment in a self selected (and presumably well motivated group of patients) remains a pertinent question.

In accordance with others (Arnold et al 2006; Lox and Freehill 1999), we showed a statistically significant difference in self-efficacy after rehabilitation, further supporting sensitivity of the CSES in the evaluation of rehabilitation. In the study of Arnold et al (1999) a generic self-efficacy tool was used and unlike the CSES test re-test reliability has not been documented.

The attainment of a personal goal can act as a powerful reinforcement of successful self-management, and could lead to a more positive appraisal of performance. Recent qualitative data shows that, in patients with Bronchiectasis, one of the perceived barriers to self management includes lack of confidence (Lavery et al 2007). Understanding more about the components and facilitators of goal attainment in rehabilitation may help us identify strategies that will support longer term behavior change and maintenance of rehabilitation benefits.

\section{Acknowledgments}

We gratefully acknowledge the support of The Health Foundation in funding this work and the statistical support of Dr Penelope Bidgood.

\section{Disclosures}

$\mathrm{RG}$ has received funding from GlaxoSmithKline, Boehringer and Pfizer. JM and FJ have no disclosures.

\section{Abbreviations}

CSES, self efficacy scale; SGRQ, st george's hospital respiratory questionnaire; BASDEC, brief assessment schedule depression cards; $\mathrm{FEV}_{1}$, forced expiratory volume in one second; FVC, forced vital capacity; LCADL, london chest activity of daily living scale.

\section{References}

American Thoracic Society (ATS). 2002. ATS statement: guidelines for the six-minute walk test. Am J Respir Crit Care Med, 166:111-7.

Arnold R, Ranchor AV, Koeter GH. et al. 2006. Changes in personal control as a predictor of quality of life after pulmonary rehabilitation, Patient Educ Couns, 61:99-108.

Arnstein P, Caudill M, Mandle CL, et al. 1999. Self efficacy as a mediator of the relationship between pain intensity, disability and depression in chronic pain patients. Pain, 80:483-91.

Bandura A. 1997. The nature and structure of self efficacy. In: Self efficacy: The exercise of control. Bandura A, (ed). New York: WH Freeman and Co.

Barnes MP, Ward AB. 2000. Textbook of rehabilitation medicine Oxford: UK. 
Bawel KJ. 2003, Exercise self-efficacy and stage of exercise behavior in older adults, Dissertation Abstracts International: Section B: The Sciences and Engineering, 63(12-B):-B.

British Thoracic Society. 2006. The Burden of Lung Disease. 2nd ed. London: British Thoracic Society.

Enright PL, Sherrill DL. 1998. Reference equations for the six-minute walk in healthy adults, Am J Respir Crit Care Med, 158:1384-7.

Ewart C. 1992. Self-efficacy: Thought control of action. Philadelphia: Taylor and Francis.

Garrod R, Ford K, Daly C, et al. 2004. Pulmonary rehabilitation: analysis of a clinical service. Physiother Res Int, 9:111-20.

Garrod R, Marshall J, Barley E, et al. 2006. Predictors of success and failure in pulmonary rehabilitation. Eur Respir J, 27:788-94.

Garrod R, Paul EA, Wedzicha JA. 2002. An evaluation of the reliability and sensitivity of the London Chest Activity of Daily Living Scale (LCADL). Respir Med, 96:725-30.

Jones F. 2006. Strategies to enhance chronic disease self-management: How can we apply this to stroke? Disabil Rehabil, 28:841-7.

Jones PW, Quirk FH, Baveystock CM, et al. 1992. A self-complete measure of health status for chronic airflow limitation. The St. George's Respiratory Questionnaire. Am Rev Respir Dis, 145:1321-7.

Kohler C L. 2002. The relationship of perceived self-efficacy to quality of life in chronic obstructive pulmonary disease. Health Psychol, 21: -614.

Lavery K, O’Neill BElborn JSReilly J, et al. 2007. Self-management in bronchiectasis: the patients' perspective. Eur Respir J, 29:541-7.

Levack WMM, Dean SG, Siegert RJ, et al. 2006, Purpose and mechanisms of goal planning in rehabilitation: The need for a critical distinction. Disabil Rehabil, 28:741-9.
Lox C, Freehill A. 1999. Impact of pulmonary rehabilitation on self-efficacy, quality of life and exercise tolerance. Rehabil Psychol, 44:208-21.

Morgan, M. D., Calverley, P. M., Clark, C., et al. 2001. British Thoracic Society Statement on Pulmonary Rehabilitation. Thorax, 56:827-34.

National Collaborating Centre for Chronic Conditions. 2004. Chronic obstructive pulmonary disease. National clinical guideline on management of chronic obstructive pulmonary disease in adults in primary and secondary care. Thorax, 59(Suppl 1):1-232.

Siela D. 2003. Use of self-efficacy and dyspnea perceptions to predict functional performance in people with COPD. Rehabil Nurs, 28:197-204.

The COPD Guidelines Group of the Standards of Care Committee of the British Thoracic Society. 1999. BTS guidelines for the management of chronic obstructive pulmonary disease. Thorax, 52:S1-S28.

Troosters T, Gosselink R, Decramer M. 2001, Exercise training in COPD: how to distinguish responders from nonresponders, $J$ Cardiopulm Rehabil, 21:10-7.

van Stel HF, Colland VT, Heins NL, et al. 2002. Assessing inpatient pulmonary rehabilitation using the patient's view of outcome. J Cardiopulm Rehabil, 22:201-10.

Waldrop D, Lightsey O, Ethington C, et al. 2001. Self-efficacy, optimism, health competence and recovery from orthopaedic surgery. J Couns Psychol, 48:233-8.

Wigal JK, Creer TL, Kotses H. 1991. The COPD Self-Efficacy Scale. Chest, 99:1193-6.

Yohannes AM, Baldwin RC, Connolly MJ. 2000. Depression and anxiety in elderly outpatients with chronic obstructive pulmonary disease: prevalence, and validation of the BASDEC screening questionnaire. Int J Geriatr Psychiatry, 15:1090-6. 